\title{
Erfolgsfaktoren im Projektmanagement
}

\author{
Heiß begehrt und doch nutzlos!?
}

\author{
Die Bestimmung von Erfolgsfaktoren ist ein zentrales Anliegen der Projekt- \\ managementforschung. Die Messbarkeit von Erfolg, aber auch die Vielfalt \\ potenzieller Erfolgsfaktoren sowie die spezifischen Rahmenbedingungen \\ von Projekten führen dieses Ziel bisweilen jedoch ad absurdum. Der Beitrag \\ zeigt die Probleme der Erfolgsfaktorenforschung auf und regt zu einem \\ reflektierten Umgang an.
}

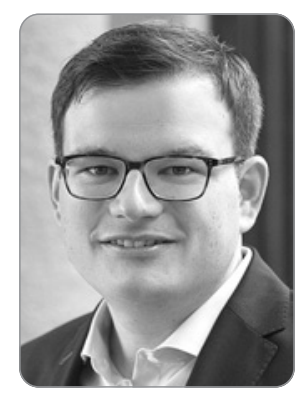

Prof. Dr. Timo Braun

ist Professor für Organisation und Management am Fachbereich Wirtschaft der Hochschule Darmstadt und assoziierter Forscher am Lehrstuhl für Unternehmenskooperation der Freien Universität Berlin. Bevorzugte Forschungsgebiete: Projekte und temporäre Organisationsformen, interorganisationale Beziehungen und Netzwerke, vernetzte Gründungsprozesse und Geschäftsmodelle.

\section{Stichwörter: Projektmanagement, Erfolgsfaktoren,} Projekterfolg, Kontingenz, Methodik

\section{Messung von Projekterfolg}

Erfolg ist naturgemäß das Ziel betriebswirtschaftlichen Handelns. Dies trifft umso mehr für das Projektmanagement zu, gibt es doch hier drei zentrale Maße des Projekterfolges. Mithilfe des sog. eisernen Dreiecks - also Qualität, Zeit und Kosten soll der Projekterfolg bestimmbar sein. Schon diese vermeintlich einfache Messung von Erfolg stößt sehr schnell an ihre Grenzen; denn Erfolg ist vielschichtig und vor allem abhängig von der Perspektive (vgl. Bea et al., 2011). Dies wird beispielsweise bei großen Infrastrukturprojekten deutlich. So ist der Bau der Elbphilharmonie in Hamburg aus reinen Projektmanagementgesichtspunkten, also mit Blick auf den Abwicklungserfolg, ein einziges Desaster. Schließlich wurden Budget und Zeit überschritten und es gab zahlreiche Mängel. Aus der Sichtweise der Tourismuswirtschaft in Hamburg erweist sich das Bauwerk demgegenüber schon jetzt als Publikumsmagnet mit internationaler Anziehungskraft, was schon jetzt auf einen großen Anwendungserfolg hindeutet. Jenseits dieser Unterscheidung von Abwicklung und Anwendung bieten sich unzählige weitere Erfolgsmaße an. Das reicht von wirtschaftlichen Indikatoren (z.B. Potenzial für Innovationen oder Folgeaufträge), über ökologische Indikatoren (z.B. Emissionen) bis hin zu Stakeholder-bezogenen Indikatoren (z.B. Mitarbeiterzufriedenheit oder gesellschaftliche Akzeptanz). Während ein Projekt aus der einen Perspektive einen bahnbrechenden Erfolg darstellt, kann das gleiche Projekt in einer anderen Dimension oder aus einer anderen Perspektive ein lupenreines Scheitern verkörpern.

\section{Bestimmung von Erfolgsfaktoren}

Die Logik des in der betriebswirtschaftlichen Praxis extrem und in der entsprechenden Forschung immer noch beliebten Erfolgsfaktorenansatzes geht nun noch einen Schritt weiter. Sie gibt sich keineswegs damit zufrieden, dass Erfolg "nur" messbar gemacht wird (diese Operationalisierung wird vielmehr schlicht vorausgesetzt). Vielmehr besteht das eigentliche Ansinnen dieses Ansatzes darin, Voraussetzungen, in der Regel gefasst als Variablen bzw. Determinanten, zu lokalisieren, die in einem kausal positiven Abhängigkeitsverhältnis zu dem wie auch immer gearteten Erfolgskonstrukt stehen. Der Erfolgsfaktorenforschung liegt die Hoffnung zu Grunde, durch die Analyse dieser potenziellen Erfolgseinflüsse den ultimativen Schlüssel zum Projekterfolg zu finden und dadurch nicht zuletzt auch Praktikern vermeintlich einfache Erfolgsrezepte als Handlungsempfehlung vorzulegen.

Potenzielle Erfolgsfaktoren gibt es dabei viele. Diese können auf sehr unterschiedlichen Ebenen angesiedelt sein (vgl. Corsten et al., 2008). So können zentrale Erfolgsfaktoren im eigentlichen Projektsystem liegen, also mit der Projektleiterin oder dem Projektleiter und deren spezifischen Kompetenzen und Fähigkeiten, dem Projektteam, der Pha- 
senplanung und -ablaufsteuerung sowie den verwendeten Projektmanagement-Tools zusammenhängen. Erfolgsfaktoren können allerdings auch im Projektkontext angelegt sein, etwa in den weiteren Rahmenbedingungen des Projektes (Technologie, Branche und Gesellschaft). Erfolgsfaktoren können zudem im engeren Projektumfeld verortet sein, beispielsweise in der Relevanz, die dem Projekt beigemessen wird, in der Komplexität oder auch im Innovationsgrad des Projektes. Nicht zuletzt können Erfolgsfaktoren auch die Sicht von Projektstakeholdern, insbesondere von Auftraggeber und Auftragnehmer, widerspiegeln und in deren jeweiligen Organisationsstrukturen, Ressourcenausstattungen und Kooperationsfähigkeiten begründet sein.

\section{Der Irrglaube an zeitlich und kontextuell stabile Erfolgsfaktoren}

Mögliche Erfolgsfaktoren gibt es also sehr viele und die situationsinvariante Gültigkeit derselben ist gelinde gesagt umstritten. In einer sehr anschaulichen, vergleichenden Fallstudie zeigt Engwall (2003), dass der Glaube an stabile, universelle Erfolgsfaktoren wenig zielführend ist. $\mathrm{Er}$ vergleicht konkret zwei Großprojekte im Bereich der Energieversorgung: ein Wasserkraftprojekt, das die Erweiterung und Renovierung eines Kraftwerks zum Gegenstand hat sowie ein Transmissionsprojekt, bei dem eine Hochspannungsverbindung durch die 0stsee ausgebaut werden soll. Beide Projekte haben einen ähnlichen Umfang von jeweils rund 250 Millionen US-Dollar und eine ähnliche Laufzeit von ca. fünf Jahren. Ein genauerer Blick auf das Wasserkraftprojekt offenbart, dass dort ein Projektmanager mit hervorragender Ausbildung und besten Kenntnissen der einschlägigen Planungs- und Steuerungstools des Projektmanagements ausgestattet ist.

Im Transmissionsprojekt ist demgegenüber ein Projektmanager eingesetzt, der einen niedrigen hierarchischen Rang ohne formale Befugnisse innehat und keinen ausdrücklichen Managementansatz verfolgt. Typische Erfolgsfaktoren (professionalisierter Projektmanagement-Ansatz, gut ausgebildeter Projektleiter etc.) würden nahelegen, dass das Wasserkraftprojekt erfolgreicher verläuft als das Transmissionsprojekt. In der Praxis war jedoch das Gegenteil der Fall. Während das Wasserkraftprojekt mit Verzögerungen zu kämpfen hatte und der Projektleiter die Kontrolle über das Projekt mehr oder weniger verlor, erwies sich das Transmissionsprojekt als eines der erfolgreichsten Projekte, das in diesem Unternehmen jemals umgesetzt wurde. Es wurde ohne größere Störungen und ohne Kostenüberschreitungen abgeschlossen.

Bei einer genaueren Analyse der Projekte nahm Engwall (2003) nun die Historizität und den relationalen Kontext der Projekte mit in den Blick. Diese Analyse offenbarte, dass das Wasserkraftprojekt lediglich eines unter vielen im Unternehmen war; es handelte sich um eine bekannte Technologie ohne spektakuläre Innovation. Zudem war der Projektleiter handverlesen vom Kunden und wurde gegen die Wünsche des Abteilungsleiters eingesetzt. Auch hatte er nur Projektmanagementerfahrung in anderen Geschäftsbereichen gesammelt, wo er zudem aus einer stärkeren Machtposition heraus agierte. Demgegenüber war das Transmissionsprojekt ein echtes Flagschiffprojekt - mit großer strategischer Tragweite und erheblichem Innovationspotenzial. So wurde beispielsweise zum ersten Mal der laufende Betrieb während der Konstruktionsarbeiten komplett aufrechterhalten. Zudem arbeiteten in diesem Projekt interorganisational eine Reihe an Partnern zusammen, die schon in Vergangenheit mehrfach erfolgreich kooperiert hatten. Der Projektleiter war seit 30 Jahren in der Abteilung tätig, zwar wenig auffällig, aber sehr geachtet.

Vor dem Hintergrund dieser Befunde stellt Engwall (2003) klar: "No Project is an island" - er meint damit, dass Projekte in all ihren Kontingenzen zu betrachten sind. Sie sind historisch eingebettet, haben also eine Vergangenheit (z.B. Vorprojektpolitik; Vorerfahrungen der handelnden Akteure miteinander), aber auch eine Zukunft (z.B. Auswirkungen von potenziellen Folgeaufträgen auf das aktuelle Handeln). Zudem laufen Projekte parallel zu anderen Ereignissen und sind eingebettet in aktuelle Rahmenbedingungen. Mit diesem Wissen erscheint es äußert fragwürdig, ob sich tatsächlich Erfolgsfaktoren identifizieren lassen, die über Zeit und Kontext hinweg stabil sind.

\section{Fundamentalkritik an der Erfolgsfaktorenforschung}

Die epistemologischen Limitationen der Erfolgsfaktorenforschung sind derart weitreichend, dass die Gültigkeit der gewonnen Erkenntnisse in Zweifel gezogen werden. Im Folgenden wird ein Überblick über die drei zentralen Argumente gegeben, die mitunter gegen die Erfolgsfaktorenforschung angeführt werden (in Anlehnung an Nicolai/Kieser, 2002):

1. Erfolgsfaktoren, die bekannt und kopierbar sind, würden im Handumdrehen ihre Wirksamkeit verlieren. Dieses Argument lässt sich leicht aus der mikroökonomischen Nullgewinnbedingung ableiten. Danach besteht zumindest in der langen Frist ein Gleichgewicht, in dem Unternehmen auf einem Markt keinen wirtschaftlichen Gewinn erzielen. Würde ein Unternehmen positive Gewinne erwirtschaften, zöge dies neue Mitbewerber in den Markt; gäbe es Verluste, würden hingegen Unternehmen aus dem Markt ausscheiden. Insofern ist damit zu rechnen, dass Erfolgsfaktoren, die hinreichend erfolgsversprechend sind, sehr schnell von Unternehmen adaptiert werden. 
2. Die Erfolgsfaktorenforschung reduziert regelmäßig die Komplexität von Praxis und entsprechender wissenschaftlicher Erkenntnis auf einfache, lineare Funktionen. Dabei ist die Auswahl der zu betrachtenden Variablen (warum sind gerade die betrachteten Faktoren relevant und nicht etwa andere, denen keine Aufmerksamkeit zu Teil wird?) ebenso fragwürdig, wie auch die unterstellten linearen Funktionen.

Beispielsweise folgen viele ökonomische Zusammenhänge keinem linearen Zusammenhang, sondern einem „inverted U-Shape“. Danach steigt der Erfolg abhängig von einem bestimmten Faktor zunächst an, erreicht aber ein Maximum und fällt bei noch stärkerem Einsatz dieses Faktors sogar ab. Ein klassisches Beispiel dafür ist das deutsche Sprichwort "viele Köche verderben den Brei“ - so sind womöglich zwei Köche in einer Küche effizienter und kreativer als ein einziger, wenn aber zu viele Köche zusammenkommen, kann das Ergebnis sich verschlechtern aufgrund von Konflikten oder weil die Köche einander regelrecht im Wege stehen.

Aber auch andere Funktionen sind denkbar, beispielsweise sprungfixe. Wenn sich beispielsweise ein Erfolgsfaktor auf einen bestimmten Ressourceneinsatz bezieht, wird sich Erfolg in manchen Fällen erst dann einstellen, wenn eine bestimmte Schwelle überschritten wird. Bis zu der Schwelle allerdings besteht kein positiver Effekt auf den Erfolg. Werden bei einem Großprojekt beispielsweise Überstunden als potenzieller Erfolgsfaktor ausgemacht, um das Projekt pünktlich abzuschließen, kann natürlich der Fall auftreten, dass trotz der Mehrarbeit ein pünktlicher Abschluss nicht erreicht wird - einfach, weil die Mehrarbeit nicht den notwendigen Umfang hatte.

3. Weiterhin ist die Untersuchung und Identifikation von Erfolgsfaktoren aus methodischer Sicht sehr problematisch, da hier viele Festlegungen bei der Wahl und Ausgestaltung des Untersuchungsdesigns zu treffen sind, die regelmäßig nicht hinreichend abgesichert werden können. Woywode (2002) hat eine ganze Reihe dieser methodischen Schwachstellen aufgezeigt. Dies sind insbesondere der sog. Key Informant Bias - so werden in wissenschaftlichen Analysen häufig die ausgewerteten Daten von einem einzigen Mitglied einer Organisation oder hier eines Projekts erhoben. Dies verkennt die Diversität entsprechender Sozialsysteme, beispielsweise verschiedene Subkulturen und Führungskonzepte in unterschiedlichen Einheiten. Ein weiterer Kritikpunkt bezieht sich auf die Historizität und Prozessualität. So unterscheiden sich Unternehmen mit Blick auf ihr Erfolgspotenzial, da sie völlig unterschiedliche Voraussetzungen mitbringen. Auch hat der frühere Erfolg oder Misserfolg einen Einfluss auf die getroffenen Maßnahmen. Tritt nach einem Misserfolg aufgrund angestoße- ner Maßnahmen eine Verbesserung auf, wird diese automatisch dem Erfolgsfaktor attribuiert. Nicht zuletzt erscheint auch problematisch, dass die Erfolgsfaktorenforschung systematisch dem Bias unterliegt nur überlebende Unternehmen zu untersuchen (",survival bias“). Interessante Erkenntnisse könnte eine genauere Analyse der Unternehmen bzw. Projekte eröffnen, die am Markt gescheitert sind - beispielsweise, ob die vermeintlichen Erfolgsfaktoren dort wirklich nicht beachtet wurden. Oft sind diese Informationen dann aber nicht verfügbar, da die Unternehmen aufgelöst wurden.

\section{Lichtblicke trotz aller Kritik?}

Die Kritik an den Erfolgsfaktoren sollte allerdings nicht darüber hinwegtäuschen, dass diese Art der Forschung (ob zu Recht oder nicht) durchaus noch ihren Platz im methodischen Repertoire der betriebswirtschaftlichen Forschung hat. Dies liegt vor allem darin begründet, dass es durchaus eine Reihe von Wissenschaftlern gibt, die den genannten Kritikpunkten eigene Argumente gegenüberstellen. So führen Homburg/Krohmer (2004) ins Feld, dass sich die Kritiker der Erfolgsfaktorenforschung "mit einer selbstentworfenen Karikatur auseinander[setzen], die in bedenklichem Ausmaß auf Pauschalisierungen sowie selektive Verwendung von Literaturquellen zurückgreift“ (ebd., S. 1). Entsprechend halten Homburg/Krohmer eine generelle Verteufelung von Erfolgsfaktoren nicht für legitim. Auch sei es nicht gerechtfertigt, Erfolgsfaktorenforscher für derart naiv bei der Generalisierung ihrer Forschungsergebnisse zu halten. Die beiden Forscher weisen zudem darauf hin, dass die Erfolgsfaktorenforschung methodisch sehr stark nachgerüstet hat, um komplexere Hypothesenstrukturen und mehrstufige Abhängigkeiten mit abbilden zu können. Ob solche komplexeren Modelle tatsächlich dafür geeignet sind, vielschichtige Kausalitäten und vor allem auch dynamische Beziehungen (z.B. Rekursionen) adäquat abbilden können, bleibt jedoch zumindest fragwürdig.

Auch speziell im Bereich des Projektmanagements hält sich die Erfolgsfaktorenforschung wacker, hat aber dennoch in den letzten zwei Jahrzehnten stark an Einfluss verloren. Sehr gut deutlich wird dies in einem Aufsatz von Söderlund (2011) der die Projektmanagementforschung in sieben Denkschulen unterteilt. Nur eine einzige davon, die „Factor School" trägt dem Ansinnen der Erfolgsfaktorenforschung Rechnung. Unter dem Strich bleiben also zahlreiche Argumente vor allem gegen die Erfolgsfaktorenforschung. Übergreifend lassen sich zwei Schlussfolgerungen zum Umgang mit der Erfolgsfaktorenforschung ableiten, die sowohl bei den Befürwortern als auch Gegnern dieser Art der betriebswirtschaftlichen Forschung auf Zustimmung treffen dürften: 
1. Sollte man sich, trotz aller methodischen Bedenken dafür entscheiden mit Erfolgsfaktoren zu arbeiten (sei es als Forschungsobjekt oder als Handlungsmaxime), darf dies niemals universell, also losgelöst von Rahmenbedingungen erfolgen.

2. Auch die Annahmen, die bei der Benennung der Erfolgsfaktoren getroffen werden, müssen stets mitgeführt und die gewonnenen Einsichten entsprechend relativiert werden.

Dies trifft gerade auch für die Projektmanagementforschung zu; denn die an Erfolgsfaktoren orientierten Ansätze haben dort über viele Jahre das Feld dominiert (vgl. Packendorff, 1995). Erst seit einigen Jahren erfreuen sich Projekte - vor allem als ein Phänomen des temporären Organisierens (vgl. dazu Braun/Sydow, 2019) - besonderen Interesses auch in der interdisziplinären Managementforschung. Hervorgehoben wird dabei, dass Projekte eine eigenständige Form von Organisation darstellen, die durch eigentümliche Strukturen, Praktiken und Mechanismen ausgezeichnet ist. Zudem erkennt dieser Forschungszweig an, dass Projekte regelmäßig Teil von größeren relationalen Strukturen und damit regelmäßig in projektbasierte oder projektunterstützte Organisationen und/oder Projektnetzwerke, aber auch in organisationale Felder eingebettet sind (vgl. Lundin et al., 2015). Eine weitere Facette sind die in Projekten angelegten Spannungsverhältnisse, etwa das zwischen Temporärem und Permanentem (vgl. Braun/Lampel, 2020). Schließlich sollen Projektergebnisse, neu generiertes Wissen und entstandene Beziehungen nicht mit dem Projektabschluss verschwinden, sondern möglichst in permanente Strukturen und Netzwerke überführt werden. Die neueren Zugänge der Projektmanagementforschung haben mithin gemeinsam, dass Projekte dort stärker als Erkenntnisobjekt und eben nicht nur als durch Tools gestaltbare Entitäten verstanden werden.

\section{Literatur}

Bea, F. X./Scheurer, S./Hesselmann, S., Projektmanagement, Konstanz 2011.

Braun, T./Lampel, J. [Hrsg.], Tensions and paradoxes in temporary organizing, Research in the Sociology of Organizations (2020, im Druck).

Braun, T./Sydow, J., Projektmanagement und temporäres Organisieren, Stuttgart 2019.

Corsten, H./Corsten, H./Gössinger, R., Projektmanagement: Einführung, München 2008.

Engwall, M., No project is an island: Linking projects to history and context, Research Policy, Vol. 32 (2003), S. 789-808.

Homburg, C./Krohmer, H., Die Fliegenpatsche als Instrument des wissenschaftlichen Dialogs. Replik zum Beitrag "Trotz eklatanter Erfolglosigkeit: Die Erfolgsfaktorenforschung weiter auf Erfolgskurs“ von Alexander Nicolai und Alfred Kieser, Universität Mannheim: Wissenschaftliche Arbeitspapiere, Nr. W74 (2004).

Lundin, R./Arvidsson, N./Brady, T./Eksted, E./Midler, C./Sydow, J. [Hrsg.], Managing and Working in Project Society - Institutional Challenges of Temporary Organizations, Cambridge 2015.

Nicolai, A./Kieser, A., Trotz eklatanter Erfolglosigkeit: Die Erfolgsfaktorenforschung weiter auf Erfolgskurs, Die Betriebswirtschaft, 62. Jg. (2002), S. 579-596.

Packendorff, J., Inquiring into the temporary organization: New directions for project management research, in: Scandinavian Journal of Management, Vol. 11 (1995), S. 319-333.

Söderlund, J., Pluralism in project management: Navigating the crossroads of specialization and fragmentation, in: International Journal of Management Reviews, Vol. 13 (2011), S. 153-176.

Woywode, W., Wege aus der Erfolglosigkeit der Erfolgsfaktorenforschung, Arbeitspaper RWTH Aachen, 2002. 